\title{
The Role of Ubiquitination and SUMOylation in Telomere Biology
}

\author{
Michal Zalzman ${ }^{1,2,3,4 *}$, W. Alex Meltzer ${ }^{1}$, Benjamin A. Portney, \\ Robert A. Brown'1 and Aditi Gupta ${ }^{1}$
}

\author{
${ }^{1}$ Department of Biochemistry and Molecular Biology, University of Maryland School of \\ Medicine, Baltimore, MD, USA. \\ ${ }^{2}$ Department of Otorhinolaryngology-Head and Neck Surgery, University of Maryland School of \\ Medicine, Baltimore, MD, USA. \\ ${ }^{3}$ Marlene and Stewart Greenbaum Cancer Center, University of Maryland School of Medicine, \\ Baltimore, MD, USA. \\ ${ }^{4}$ The Center for Stem Cell Biology and Regenerative Medicine, University of Maryland School of \\ Medicine, Baltimore, MD, USA. \\ *Correspondence: mzalzman@som.umaryland.edu \\ https://doi.org/10.21775/cimb.035.085
}

\begin{abstract}
Telomeres are a unique structure of DNA repeats covered by proteins at the ends of the chromosomes that protect the coding regions of the genome and function as a biological clock. They require a tight regulation of the factors covering and protecting their structure, as they are shortened with each cell division to limit the ability of cells to replicate uncontrollably. Additionally, they protect the chromosome ends from DNA damage responses and thereby, prevent genomic instability. Telomere dysfunction can lead to chromosomal abnormalities and cancer. Therefore, dysregulation of any of the factors that regulate the integrity of the telomeres will have implications to chromosomal stability, replicative lifespan and may lead to cell transformation. This review will cover the main factors participating in the normal function of the telomeres and how these are regulated by the ubiquitin and SUMO systems. Accumulating evidence indicate that the ubiquitin and SUMO pathways are significant regulators of the shelterin complex and other chromatin modifiers, which are important for telomere structure integrity. Furthermore, the crosstalk between these two pathways has been
\end{abstract}

reported in telomeric DNA repair. A better understanding of the factors contributing to telomere biology, and how they are regulated, is important for the design of new strategies for cancer therapies and regenerative medicine.

\section{Telomere structure and function}

Telomeres are DNA structures covered by proteins at the ends of the chromosomes that serve several key biological functions. Primarily, they function as a 'biological clock' that regulates the replicative lifespan of cells, as well as protect the integrity of the ends of the chromosomes from nucleolytic digestion (Vaziri et al., 1994; Vaziri and Benchimol, 1996; Karlseder et al., 1999; O'Sullivan and Karlseder, 2010). The telomeres consist of several components that cooperate to mediate telomere function: A DNA repeat sequence (TTAGGG in mammals), the Shelterin complex, a group of proteins that function to cover and compact the repeat sequences and interacting RNA components. Telomere length varies between organisms ranging from a few hundred base pairs in yeast, to tens of kilobase pairs in mammals. In humans, the length of 
the telomere can range anywhere from $5 \mathrm{~kb}-20 \mathrm{~kb}$ (Samassekou et al., 2010).

Telomeres play a critical role in cellular replicative lifespan and protect the coding regions of the genome. Therefore, dysfunctions or disruptions of those nucleoprotein structures at the end of the chromosomes can present as serious pathologies. These telomere syndromes span several different disease areas including blood, lung and liver disease, bone marrow failure, age related disease, and cancer. Collectively, telomere erosion has a strong correlation with several different organ specific diseases. Understanding the mechanisms that regulate telomere length will help guide diagnosis, prevention and treatment.

\section{The Hayflick limit and the end replication problem}

The telomere's ability to act as a biological clock serves as a powerful mechanism for tumour suppression. Primary cells can potentially divide a limited amount of times before reaching a state where they can no longer replicate (Hayflick and Moorhead, 1961). This replication limit, called the Hayflick limit, was first described by Dr Leonard Hayflick in 1961. In this work, he and Dr Paul Moorhead demonstrated that unlike cancer cells, primary cells age in culture and eventually die, disproving a longstanding theory that all cell lines in culture are immortal (Hayflick and Moorhead, 1961). In the next decade, the connection between cellular ageing and telomeres was hypothesized to be a result of the 'end replication problem', which is a by-product of the linear nature of eukaryotic DNA. DNA polymerase can only synthesize DNA in the $5^{\prime}-3^{\prime}$ direction to generate new DNA. Replication of the leading 5'-3' DNA strand allows DNA polymerase to generate a complete complimentary strand. Conversely, the $3^{\prime}-5$ ' lagging DNA strand, requires the activity of the enzyme Primase which adds RNA primers for the creation of 100-200 base pair sized DNA fragments, called the Okazaki fragments. This allows DNA polymerase to synthesize DNA in the 5'- 3' direction. Following replication, the RNA primers are removed and the Okazaki fragments are ligated together. However, Primase cannot add RNA primers at the end of the lagging strand. This issue leads to an incomplete replication, creating a 75-300 nucleotide long overhang of the

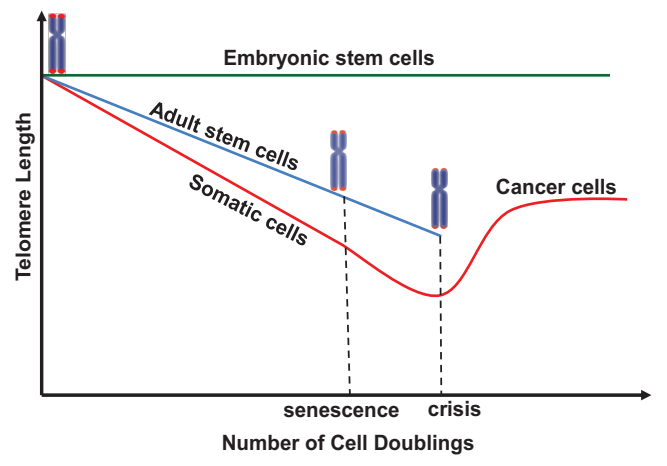

Figure 16.1 Telomere length decreases as cells age. In culture, every cell division results in loss of telomeric DNA. The rate of telomere shortening varies between cell types and continues until the telomeres reach a point when they are short enough to induce a signal to enter into senescence, i.e. growth arrest. Further telomere erosion and in vivo clearance by the immune system leads to apoptosis or culture crisis.

3 ' telomeric end (Makarov et al., 1997; McElligott and Wellinger, 1997; Chai et al., 2006). To protect this overhang, proteins are recruited for the further processing needed to properly create a protected DNA structure called a T-loop, resulting in overall resection of the end of the chromosome. Thus, as cells divide and DNA is replicated, the telomeres gradually shorten (Fig. 16.1). At a certain predetermined length, which varies between cell types, the telomeres become critically short and signal for the cell to cease further replication, inciting cellular senescence or death.

\section{Telomeres and protecting the genome}

The ability of cells to detect and repair DNA damage is a powerful mechanism for cellular maintenance and cancer prevention. One such trigger for this response is via the detection of exposed linear DNA (Chapman et al., 2012). Several cellular responses to detection of broken DNA exist, including repair of fragments, cell cycle arrest, or, if the damage is severe, apoptotic cell death (Jackson and Bartek, 2009). These mechanisms have evolved to prevent aberrant genomic instability resulting in mutations that can lead to cancer. Paradoxically, because mammalian DNA is linear, and due to the 3 ' overhang, telomeres can be recognized as DNA breaks by the DNA repair machinery and damage 
response agents, unless properly shielded. Therefore, to protect the telomeres, the repeat sequences fold into themselves to create a 'T-loop' structure to avoid recognition double-stranded DNA damage. The repeat sequence is masked by a network of proteins called the shelterin complex (Fig. 16.2).

\section{T-loops}

A displacement loop or a D-loop, is a DNA structure in which a double-strand of DNA is additionally occupied by a third single-stranded DNA based on base complementarity. In the context of the telomere, this structure is referred to as a T-loop and is created through the 3 ' strand invasion of the G-rich overhang, created during DNA replication (Greider, 1999; Griffith et al., 1999; Murti and Prescott, 1999; de Bruin et al., 2000; Muñoz-Jordán et al., 2001). The displacement occurs at a distant place from the end of the telomere, creating a large duplex lariat structure (de Lange, 2005). Evidence for these structures first arose from work done in vitro, demonstrating that artificially generated telomeres form large loops only in the presence of a 3 ' overhang (Greider, 1999). This looped structure shelters the exposed G-rich overhang, caused by the end replication problem, and in the process protects the C-rich shortened end of the telomere. This triplex nucleic acid structure allows DNA repair proteins to distinguish between breaks in the
DNA and the telomeric end, successfully blocking any DNA repair response (Fig. 16.2).

\section{The shelterin complex}

To properly form and conserve the T-loop structure, a number of proteins are required, collectively called the shelterin complex (Fig. 16.2). The shelterin complex is comprised of six proteins: TRF1 (telomere repeat binding factor 1) (Zhong et al., 1992; Chong et al., 1995), TRF2 (telomere repeat binding factor 2) (Bilaud et al., 1997), POT1 (protection of telomeres 1) (Baumann and Cech, 2001), TIN2 (TRF1-interacting nuclear protein 2) (Kim et al., 1999), RAP1 (repressor and activator protein 1) (Li et al., 2000), and TPP1 (POT1-and TIN2interacting protein) (Houghtaling et al., 2004).

The shelterin complex protects the telomeres in multiple ways. First, through facilitating the T-loop formation by promoting the strand invasion of the 3' DNA overhangs. This prevents the detection of the exposed single-stranded DNA, and therefore blocks the detection as DNA break (Griffith et al., 1999). Both TRF2 and TRF1 are able to remodel artificial telomeres in vitro to create the T-loop structures (Bianchi et al., 1997; Griffith et al., 1999; Stansel et al., 2001). Further, TIN2 acts to enhance the TRF1 mediated T-loop formation (Kim et al., 2003). Secondly, the shelterin complex interacts with and inhibits the DNA damage response

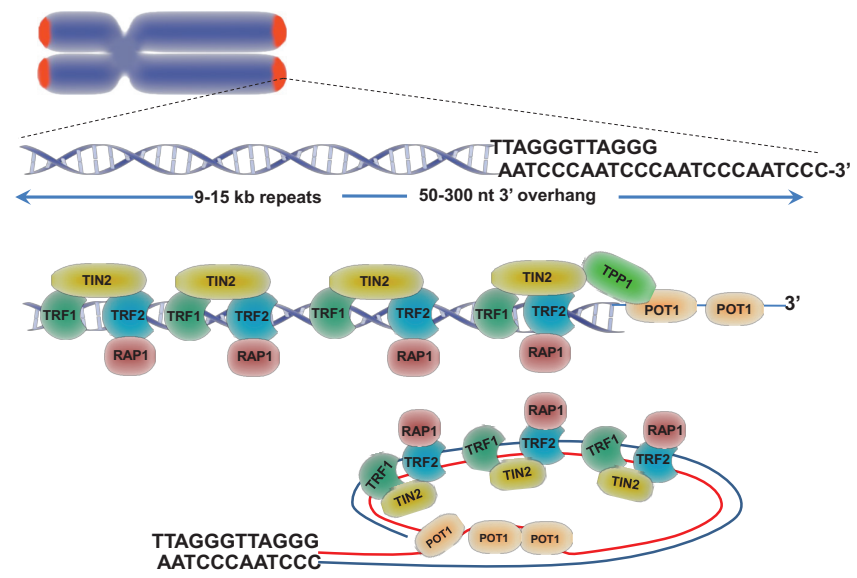

Figure 16.2 Telomere structure. Telomeres are structures at the ends of the chromosomes that contain 6-nucleotide repeat sequences (top). Incomplete replication of the lagging strand results in a G-Rich $3^{\prime}$ overhang (middle). The repeat sequences are coated with a cluster of proteins called the shelterin complex. The shelterin complex helps loop the G-rich overhang into the double-strand telomere sequence, creating a T-loop (bottom). 
pathway (Karlseder et al., 2004). A deficiency of any of the shelterin complex components leads to telomere deprotection, genomic instability and potentially to cellular senescence. When TRF2 is down-regulated, the ATM kinase pathway is activated, leading to cell cycle arrest (Karlseder et al., 1999). DNA damage signalling is also seen in the absence of TIN2 or POT1 (Kim et al., 2004; Hockemeyer et al., 2005). Lastly, the shelterin complex is thought to inhibit the telomere maintenance enzyme, telomerase, which can add telomere repeats lost during cell replication (Loayza and de Lange, 2003; Liu et al., 2004; Kelleher et al., 2005; Lei et al., 2005).

\section{Protein turnover}

Concentrations and spatial gradients of proteins must be able to rapidly respond to extracellular cues and cell status (Korolchuk et al., 2010). Even subtle protein imbalances can drastically impact important cellular processes. Therefore, the regulation of protein degradation and turnover play an important role in the cell-life cycle (Ciechanover, 2005). Protein levels in the cells are at a constant state of turnover. Continuous synthesis of proteins and degradation is required for steady state protein levels and cellular homeostasis (Reinstein and Ciechanover, 2006). Hence, protein turnover plays an important role in regulating cellular fitness (Ciechanover, 2005). The balance is maintained through three major systems regulating the maintenance of proper protein folding and native conformation. The first is the chaperone system, which includes stress-induced heat shock proteins (HSPs) involved in protein folding. The second is the ubiquitin-proteasome system (UPS), controlling the degradation and clearance of misfolded proteins. Finally, the autophagy system, responsible for the recycling and degradation of long lived, structural proteins and organelles. (Eskelinen and Saftig, 2009; Yang and Klionsky, 2010).

Protein turnover, or degradation, influences a variety of basic cellular functions. Another primary role of protein degradation is to serve as an intracellular quality control system through elimination of misfolded or damaged proteins. Accumulations of misfolded proteins can create non-physiological interactions with other proteins that are particularly harmful to the cell. Proteins can be damaged in multiple ways, including genetic mutation, misfolding in the ER, translational errors, toxic factors from the environment, or intracellular toxic agents resulting from ageing or disease. Damaged proteins must either be quickly repaired or eliminated in order to prevent further harm to the cell (Goldberg, 2003). More recently, regulated protein degradation has been shown to control complex cellular processes including metabolism, cell cycle, transcription, signal transduction and apoptosis (Ciechanover, 2005; Chen and Sun, 2009).

Owing to the amount of vital cellular functions influenced by protein turnover, it is not surprising that dysfunction in protein degradation has been implicated in multiple diseases (Reinstein and Ciechanover, 2006). Aberrant protein stabilization or accelerated degradation of proteins changes their steady-state levels, precipitating disease. Neurological disorders such as Parkinson's disease are highly linked to protein turnover dysfunction. In many cases, aggregates of disease specific proteins are accumulated and cannot be degraded (Ciechanover and Brundin, 2003; Tanaka et al., 2004). Disruptions in protein turnover have also been identified in cancer, where stabilization of oncogenes and destabilization of tumour suppressors contribute to malignancy (Ohta and Fukuda, 2004).

Proteins have developed specialized functions and are therefore degraded at widely different rates. The standard measurement is also known as protein half-life (Zhou, 2004; Hinkson and Elias, 2011). Protein half-lives can range from just a few minutes to hours, in the case of transcription factors and regulatory proteins, to up to multiple days for structural proteins (Goldberg, 2003). In order to control the various cellular functions regulated by protein turnover, cells must precisely control protein half-lives.

\section{The function of the ubiquitin- proteasome system and the process of SUMOylation}

The Ubiquitin-Proteasome System (UPS) is a highly regulated apparatus responsible for intracellular protein turnover and degradation (Hershko and Ciechanover, 1998; Nandi et al., 2006). The UPS is a selective process orchestrated by a series of ubiquitin ligase enzymes specific to the pathway. The role of the UPS has been demonstrated in the 
A.

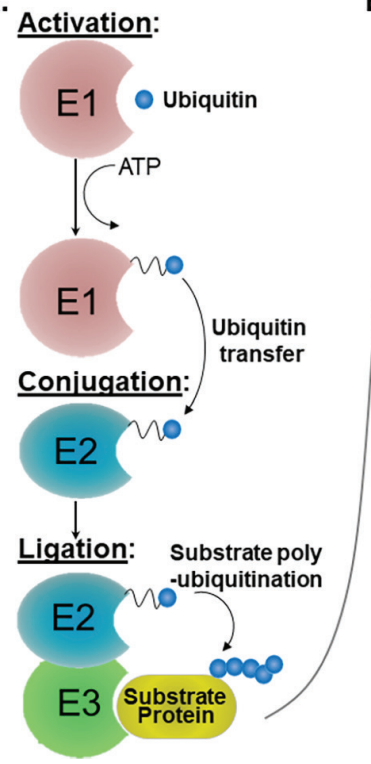

B.

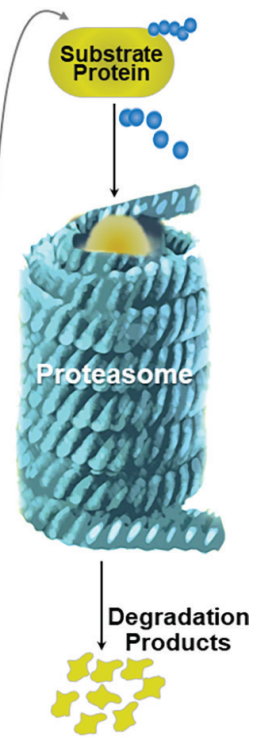

Figure 16.3 The ubiquitin proteasome system. (A) Illustration of the ubiquitination process. (B) Polyubiquitinated protein is degraded by the proteasome.

turnover of up to $90 \%$ of all cellular proteins (Huang and Figueiredo-Pereira, 2010). As the name Ubiquitin suggests, the UPS is involved in the regulation of a wide array of biological processes including antigen presentation, DNA repair, protein trafficking, epigenetic regulation, and the cell cycle (Nandi et al., 2006; Al-Hakim et al., 2010). The proteasome has become a drug target, as fluctuations in proteasomal activity and defects in function have been linked to a variety of diseases (Dahlmann, 2007; Bedford et al., 2011).

The ubiquitin gene encodes for a small, 76 amino acid protein. Ubiquitin is covalently conjugated to lysine residues of substrate proteins through an isopeptide linkage in a post-translational process called ubiquitination (Pickart and Eddins, 2004). Ubiquitination occurs through an enzymatic cascade in three steps: activation, conjugation, and ligation. Each step is facilitated by a distinct ubiquitin enzyme (Fig. 16.3A,B). The initial step, activation of the ubiquitin molecule by an ATP dependent E1 ubiquitin enzyme, produces a ubiquitin-adenylate intermediate. Next, the activated ubiquitin is transferred to the active site of an E2 ubiquitin enzyme via a trans(thio)esterification reaction (Ye and Rape, 2009). In the final ligation

step, E3 ubiquitin enzymes facilitate the transfer, either directly (HECT domain E3s) or indirectly (RING domain E3s), of the ubiquitin molecule from the E2 enzyme to a lysine residue in the substrate protein. Successive rounds of the ubiquitination process result in ubiquitin chains (Callis, 2014). The specificity of ubiquitination increases with each step, as only two genes are responsible for E1 ubiquitin enzymes and only 35 genes for E2 enzymes. However, there are well over six hundred E3 ubiquitin ligases that recognize target substrates, thereby conferring specificity to the UPS (Ardley and Robinson, 2005).

E3 ubiquitin ligases fall into two main structural families that differ in how ubiquitin is transferred from E2 enzyme to substrate: the HECT (Homologous to the E6-AP Carboxyl Terminus) domain and the RING domain (Really Interesting New Gene) ligases (Buetow and Huang, 2016). HECT domain E3 ligases contain a catalytic cysteine residue that accepts the ubiquitin molecule from the E2 ligase, forming a thioester intermediate. It is the HECT domain E3 ligase that then directly transfers the ubiquitin to the substrate (Morreale and Walden, 2016). Approximately 30 HECT domain E3 ligases have been identified. Alternatively, ubiquitination with RING domain E3 ligases is facilitated by E2 ligases. The RING E3 ligase simply acts as a scaffold between the E2 ligase and substrate (Ozkan et al., 2005). RING domain E3 ligases are the predominant family in mammals, with over 300 enzymes identified.

The Small Ubiquitin-like Modifier (SUMO) system regulates protein function by covalently attaching and detaching small protein chains that are analogous to ubiquitin. The enzymatic cascade of SUMOylation is similar to that involved in ubiquitination. Like the ubiquitin system, SUMOylation is involved in multiple cellular processes, such as regulation of transcription, apoptosis, transport to the nucleus, protein stability, cellular stress response and cell cycle progression [1]. The last four amino acids of the C-terminus of SUMO are cleaved off allowing the formation of an isopeptide bond between the $\mathrm{C}$-terminal glycine residue and an acceptor lysine of the target protein. There are four SUMO proteins known as SUMO1-4. Similar to ubiquitination, SUMOylation is regulated by E1 activating enzymes, E2 conjugation enzymes, E3 SUMO ligases and SUMO specific proteases 
which are involved in the removal of SUMO conjugates. There are a number of diseases associated with SUMOylated proteins, such as Parkinson disease (PD) and Alzheimer's disease. Histone SUMOylation was first identified in 2003. Histone 4 can be modified by SUMO through the HDAC. However, unlike ubiquitin, SUMOylation does not directly mark protein for proteasomal degradation, but often works to induce the function or shift the localization of the modified protein in the cell.

\section{Telomere regulation in cancer}

Cancer is considered a disease state in which dysfunctional cells replicate at a high rate and lose their ability to interact properly with their environment, resulting in abnormal growth and invasion into nearby and distant tissues. In 2016, an estimate of 1.6 million new cases of cancer were diagnosed, in the U.S. with roughly 600,000 deaths from the disease, making it the second leading cause of death (Jemal et al., 2017). Cancer is thought to develop in a multistep process of sequential rounds of genetic mutations that convert a normal cell into a malignant cell (Jonkers, 2012). However, in addition to mutation accumulation, a second crucial event must occur in order to ensure unlimited cell replication. Otherwise, a mutated cell will age and cease to divide before it can be detected as a tumour. This event must lead to the evasion or the reversal of telomeres shortening and physiological ageing during cell division (Sugimoto et al., 2004; Smith et al., 2016). Telomere dysfunction is further linked to cancer, as patients with telomere related diseases have an increased risk for developing cancer (de la Fuente and Dokal, 2007; Alter et al., 2009; Diaz de Leon et al., 2010; Alder et al., 2011). This is likely cause by shortened telomeres and faulty repair mechanisms that trigger chromosomal fusions and increase genomic instability.

\section{Telomerase}

Telomerase is a ribonucleoprotein complex that consists of an RNA component (TERC) and a reverse transcriptase (TERT). TERC provides the RNA template required for the reverse transcription activity of the enzyme TERT. Together with additional complex components, they add repeats to shortened telomeres. Telomerase activity is most robust during embryonic development, and then persists in much lower levels in certain human adult tissues (Broccoli et al., 1995; Counter et al., 1995; Härle-Bachor and Boukamp, 1996; Schieker et al., 2004). Telomerase activity and the resulting telomere elongation can lead to the bypass of replicative senescence and to cell immortalization (Garbe et al., 2014; Smith et al., 2016). Expectedly, mutations in telomerase complex components are commonly found in telomere syndromes such as dyskeratosis congenita and aplastic anaemia (de la Fuente and Dokal, 2007; Savage and Alter, 2009; Diaz de Leon et al., 2010; Dokal, 2011; Nelson and Bertuch, 2012).

The ability to maintain telomere length in cancer cells is traditionally attributed to the enzyme telomerase (Kunická et al., 2008). Telomerase is expressed in $85 \%$ of all cancers. The remaining telomerase negative cancers must activate other mechanisms to maintain telomere integrity which are collectively called: Alternative Lengthening of Telomeres (ALT) mechanisms (Bryan et al., 1997; Cesare and Reddel, 2010). While it has been shown that ALT can still function in the presence of telomerase overexpression, it is generally assumed that these mechanisms act in a mutually exclusive manner (Cerone et al., 2001; Grobelny et al., 2001; Perrem et al., 2001). In fact, inhibition of telomerase by a drug can ultimately lead to resistance through activation of ALT (Hu et al., 2012; Hu et al., 2016).

\section{Alternative lengthening of telomeres (ALT)}

The less understood mechanism of telomere maintenance in cancer is alternative lengthening of telomeres (ALT). The defining characteristic of the canonical ALT mechanism is its independence from telomerase activity. ALT dependent cancer cells also have other distinguishing characteristics, including extrachromosomal circular telomeric DNA (c-circles) (Cesare and Griffith, 2004; Henson et al., 2009), telomeric DNA associated with promyelocytic leukaemia (PML) bodies (Yeager et al., 1999), heterogeneous telomere lengths across different chromosomes, and increased telomere recombination events (Bailey et al., 2004). Unlike telomerase, that uses an RNA template, cancers that present with canonical ALT are thought to use telomeric DNA as a template for extension. The 
template can be a sister chromatid strand (sister chromatid exchange), extrachromosomal circular telomeric DNA, or a telomeric sequence from a separate chromosome (homologous recombination) (Cesare and Reddel, 2010; Yu et al., 2014). Therefore, proteins involved in homologous recombination (HR) were shown to be required for successful telomere maintenance in ALT (Zhong et al., 2007).

\section{Ubiquitination-mediated regulation of the shelterin}

The Shelterin complex caps the telomeres and acts as a protective layer covering the telomeric DNA at end of the chromosomes. Shelterin is composed of multiple proteins that have been shown to be regulated by the ubiquitin-proteasome system. The Ubiquitin-mediated degradation of the Shelterin component TRF1 is the first example and is facilitated by three E3 ligases: RLIM, FBX4 and $\beta$-TRCP1 (Lee et al., 2006; Her and Chung, 2009; Wang et al., 2013). RLIM targets telomere DNAbound TRF1 for proteasomal degradation (Her and Chung, 2009). Conversely, FBX4 binds to the $\mathrm{N}$-terminal region of the dimerization domain of unbound TRF1 and targets it for degradation (Lee et al., 2006). Consequently, when either of these enzymes is depleted, TRF1 levels are stabilized, causing telomerase inhibition and leading to a decrease in telomere length and to impaired cell growth. Furthermore, TRF1 levels are also indirectly and independently regulated by the factors U2AF65 (Kim and Chung, 2014), TIN2 (Ye and de Lange, 2004), and the F-box protein $\beta$-TRCP1 (Wang et al., 2013), which were shown to positively regulate of TRF1 by acting as competitive inhibitors to FBX4, and physically preventing its interaction with TRF1 and subsequent ubiquitinmediated degradation. TIN2 itself acts as part of the shelterin complex, therefore, further regulation of TIN2 turnover is achieved by the ubiquitin system as the interaction with the E3 ligase SIAH2 sends it to proteasomal degradation (Bhanot and Smith, 2012).

The turnover of another important shelterin subunit, TRF2, has also been shown to be regulated by ubiquitination. With either normal cell replication or telomere dysfunction, telomere shortening leads to reduced levels of TRF2 binding and as a result, to a loss of TRF2 mediated telomere protection. Consequently, a cascade of events is triggered in the cell. First, the ATM kinase is activated which in turn phosphorylates the tumour suppressor p53. Then, the activated p53 triggers replicative senescence. Additionally, as a feedback loop, p53 induces the transcription of the E3-ubiquitin ligase SIAH1, which targets TRF2 for degradation. Finally, this cascade is further amplified, as a positive feedback loop, with increased p53 activation leading to further increased SIAH1 levels and TRF2 ubiquitination (Fujita et al., 2010).

Another shelterin subunit regulated by ubiquitin-mediated degradation is TPP1. Although the E3 ubiquitin ligases for TPP1 are still unknown, it has been shown that inhibition of the proteasome system leads to stabilization of TPP1 protein levels. The human TPP1 levels are shown to be further regulated by interaction with the deubiquitinating enzyme USP7 which removes ubiquitin chains from it (Zemp and Lingner, 2014). In mice, the E3 ligase RNF8, ubiquitinates TPP1 and is required for its stabilization at telomeres (Rai et al., 2011). However, the role of ubiquitination of human TPP1 still remains to be discovered, as it has not been demonstrated to affect its function, or its interaction with other shelterin components such as TIN2, POT1 or its interaction with telomerase (Zemp and Lingner, 2014).

\section{SUMOylation-mediated regulation of the shelterin}

Crosstalk between SUMOylation and ubiquitination were also to contribute to regulation and turnover of TRF2. The E3 SUMO ligase PIAS1 interacts with and was shown to SUMOylate TRF2. This allows the interaction of the SUMO-targeted ubiquitin ligase RNF4 which in turn ubiquitinates TRF 2 and send it to degradation by the proteasome and by that contributes to TRF2 turnover without affecting telomere integrity (Her et al., 2015).

Finally, SUMOylation was also shown to tag Rap1 in the budding yeast, Saccharomyces cerevisiae. The SUMO-targeted ubiquitin ligase Uls1 binds SUMOylated Rap1, ubiquitinates it and send it to proteasome mediated degradation. Loss of Uls1 results in accumulation of poly-SUMOylated Rap1 and leads to telomere fusions. Elimination of Rap 1 SUMOylation sites in Uls1-depleted cells prevents 
telomere fusion suggesting that poly-SUMOylated Rap1 is non-functional in telomere protection from NHEJ (Lescasse et al., 2013). In mammals, the shelterin complex component RAP 1 forms a dimer with TRF2 to protect the telomeres from inappropriate processing by the homologous recombination pathway and from rapid telomere resection, which would otherwise result in telomere loss and fusions in both mouse and human cells (Rai et al., 2016).

An additional role for SUMOylation in telomere length regulation through shelterin modulation has been shown in fission yeast (Miyagawa et al., 2014). SUMOylation of Tpz1, the fission yeast homologue of TPP1, is required to maintain telomere length. The mechanism was elucidated whereby SUMOylated Tpz1 recruits Stn-Ten1 to the telomere, which in turn inhibits the binding of telomerase and telomere replication. Mutation of the Lysine 242 on Tpzl prevents its SUMOylation, prevents Stn-Ten 1 recruitment, and results in abnormally long telomeres. These findings have yet to be demonstrated in mammalian systems, however, the CST complex (including STN and TEN1) are well conserved in humans and inhibit telomerase activity (Chen et al., 2012) suggesting the mechanism may be similar. In summary, the binding of shelterin complex subunits to the telomeric DNA is essential for telomere integrity and genome stability, but it relies heavily on the proper regulation of the Shelterin by the ubiquitin and SUMO systems.

\section{Telomere chromatin regulation by ubiquitin}

Post translation modification of histones tails such as methylation and acetylation are a powerful means for chromatin structure modulation. In yeast (Saccharomyces cerevisiae), the E2 Ubiquitin ligase Rad6 (Ubc2) was shown to regulate histone H3 methylation at lysine 4 (H3K4-me) through addition of one molecule of ubiquitin to histone $\mathrm{H} 2 \mathrm{~B}$ (mono-ubiquitination of H2B) at Lys 123. Furthermore, a mutation abolishing the Lysine 123 in $\mathrm{H} 2 \mathrm{~B}$ leads to telomere transcription and the expression of the long non-coding RNA TERRA (Sun and Allis, 2002).

Another important modulator of telomere chromatin state by histone $\mathrm{H} 2 \mathrm{~A}$ ubiquitination is mediated through the E3 ligase RNF8. RNF8 is DNA-damage-responsive protein that mediates histone ubiquitination signalling and plays a critical role in the cellular response to genotoxic stress and DNA damage repair (Huen et al., 2007). However, it was further shown to affect telomere stability and facilitate telomere fusion by ubiquitination of histone $\mathrm{H} 2 \mathrm{~A}$ and $\mathrm{H} 2 \mathrm{AX}$. Consistent with the critical effect of RNF8 on uncapped telomeres, loss of RNF8, as well as of the E3 ligase RNF168, reduces telomere-associated genome instability. These data suggest that $\mathrm{H} 2 \mathrm{~A}$ mono-ubiquitination may enhance cancer development by facilitating telomere fusion and dysfunction (Peuscher and Jacobs, 2011) and highlight mono-ubiquitination in the maintenance of telomere integrity.

Poly-ubiquitination has also been shown to be important for telomere biology. The early embryonic gene Zscan4 (Zinc finger and SCAN domain containing 4) promotes genomic stability and telomere homeostasis in mouse embryonic stem (ES) cells (Zalzman et al., 2010). Zscan4 is transiently expressed (Zalzman et al., 2010), with protein level bursts associated with chromatin remodelling (Amano et al., 2013; Akiyama et al., 2015) and nuclear reprogramming during the generation of induced pluripotent stem (iPS) cells (Hirata et al., 2012; Jiang et al., 2013; Park et al., 2015). The human ZSCAN4 has been shown to interact with shelterin complex components (Lee and Gollahon, 2014,2015 ), and has been suggested to play a role in cancer (Zalzman et al., 2010; Lee and Gollahon, 2014; Portney et al., 2018). Given the important role of ZSCAN4 and its transient expression in the cell (Falco et al., 2007; Zalzman et al., 2010), maintaining the delicate balance between its protein synthesis and degradation is critical for stem cell and potentially cancer cell function. Therefore, stringent regulation of the levels of ZSCAN4 is required to effectively control its function. Indeed, ZSCAN4 protein degradation was shown to be regulated by the ubiquitin-proteasome system (Portney et al., 2018). The E3 ubiquitin ligase RNF20 negatively regulates ZSCAN4 protein by poly-ubiquitination. Further, RNF20 depletion does not affect ZSCAN4 RNA transcription, yet it leads to the accumulation and stabilization of ZSCAN4 protein, suggesting it as a negative regulator of ZSCAN4 protein stability. Due to the important role of ZSCAN4 in the 
generation of iPS cells, these data have important implications for role of ubiquitination in the regulation of telomere and genomic stability (Portney et al., 2018).

\section{Discussion and perspectives}

The function of the telomeres as a biological clock requires a tight regulation of the factors covering and protecting their structure, in order to limit the ability of cells to replicate uncontrollably. Telomeres also protect the chromosome ends from activating DNA damage responses and thereby, prevent chromosomal fusions and genomic instability. Telomere dysfunction leads to increased chromosomal abnormalities and cancer development (Feldser et al., 2003; Blasco, 2005; Gilley et al., 2005). Consequently, a dysregulation of any of the factors that regulate telomere structure integrity and length will cause major implications to chromosomal integrity, cellular lifespan and cancer transformation. A better understanding of the process and factor controlling telomere processing is important for the development of new strategies for cancer therapies and regenerative medicine. Compelling evidence suggest that the ubiquitin and SUMO pathways are important regulators of both the shelterin and telomere chromatin structures. Moreover, these posttranslational modifications contribute to the cellular response to damaged telomeres. Further research is needed to promote our understanding of the effect of these modifications on telomere regulation and function and their significance to human health. Likewise, additional studies are needed to determine the underlying mechanisms, by which Ubiquitination and SUMOylation regulate telomere factors and protect the genome. The crosstalk between the two pathways has been demonstrated in both genomic and telomeric DNA repair. Further research will elucidate and the modifications unique to telomere maintenance and repair, which may allow to device new strategies for targeting the telomeres without triggering unwanted mechanisms for genomic DNA repair.

\section{References}

Akiyama, T., Xin, L., Oda, M., Sharov, A.A., Amano, M., Piao, Y., Cadet, J.S., Dudekula, D.B., Qian, Y., Wang, W., et al. (2015). Transient bursts of Zscan4 expression are accompanied by the rapid derepression of heterochromatin in mouse embryonic stem cells. DNA Res. 22, 307-318. https://doi.org/10.1093/dnares/ dsv013.

Alder, J.K., Cogan, J.D., Brown, A.F., Anderson, C.J., Lawson, W.E., Lansdorp, P.M., Phillips, J.A., Loyd, J.E., Chen, J.J., and Armanios, M. (2011). Ancestral mutation in telomerase causes defects in repeat addition processivity and manifests as familial pulmonary fibrosis. PLOS Genet. 7, e1001352. https://doi.org/10.1371/journal. pgen. 1001352.

Al-Hakim, A., Escribano-Diaz, C., Landry, M.C., O’Donnell, L., Panier, S., Szilard, R.K., and Durocher, D. (2010). The ubiquitous role of ubiquitin in the DNA damage response. DNA Repair 9, 1229-1240. https://doi. org/10.1016/j.dnarep.2010.09.011.

Alter, B.P., Giri, N., Savage, S.A., and Rosenberg, P.S. (2009). Cancer in dyskeratosis congenita. Blood 113, 6549-6557.

Amano, T., Hirata, T., Falco, G., Monti, M., Sharova, L.V., Amano, M., Sheer, S., Hoang, H.G., Piao, Y., Stagg, C.A., et al. (2013). Zscan4 restores the developmental potency of embryonic stem cells. Nat. Commun. 4, 1966. https://doi.org/10.1038/ncomms2966.

Ardley, H.C., and Robinson, P.A. (2005). E3 ubiquitin ligases. Essays Biochem. 41, 15-30.

Bailey, S.M., Brenneman, M.A., and Goodwin, E.H. (2004). Frequent recombination in telomeric DNA may extend the proliferative life of telomerase-negative cells. Nucleic Acids Res. 32, 3743-3751. https://doi.org/10.1093/ nar/gkh691.

Baumann, P., and Cech, T.R. (2001). Pot1, the putative telomere end-binding protein in fission yeast and humans. Science 292, 1171-1175. https://doi. org/10.1126/science.1060036.

Bedford, L., Lowe, J., Dick, L.R., Mayer, R.J., and Brownell, J.E. (2011). Ubiquitin-like protein conjugation and the ubiquitin-proteasome system as drug targets. Nat. Rev. Drug Discov. 10, 29-46. https://doi.org/10.1038/ nrd3321.

Bhanot, M., and Smith, S. (2012). TIN2 stability is regulated by the E3 ligase Siah2. Mol. Cell. Biol. 32, 376-384. https://doi.org/10.1128/MCB.06227-11.

Bianchi, A., Smith, S., Chong, L., Elias, P., and de Lange, T. (1997). TRF1 is a dimer and bends telomeric DNA. EMBO J. 16, 1785-1794. https://doi.org/10.1093/ emboj/16.7.1785.

Bilaud, T., Brun, C., Ancelin, K., Koering, C.E., Laroche, T., and Gilson, E. (1997). Telomeric localization of TRF2, a novel human telobox protein. Nat. Genet. 17, 236-239. https://doi.org/10.1038/ng1097-236.

Blasco, M.A. (2005). Telomeres and human disease: ageing, cancer and beyond. Nat. Rev. Genet. 6, 611-622. https://doi.org/10.1038/nrg1656.

Broccoli, D., Young, J.W., and de Lange, T. (1995). Telomerase activity in normal and malignant hematopoietic cells. Proc. Natl. Acad. Sci. U.S.A. 92, 9082-9086.

Bryan, T.M., Englezou, A., Dalla-Pozza, L., Dunham, M.A., and Reddel, R.R. (1997). Evidence for an alternative mechanism for maintaining telomere length in human tumors and tumor-derived cell lines. Nat. Med. 3, 1271-1274. 
Buetow, L., and Huang, D.T. (2016). Structural insights into the catalysis and regulation of E3 ubiquitin ligases. Nat. Rev. Mol. Cell Biol. 17, 626-642. https://doi. org/10.1038/nrm.2016.91.

Callis, J. (2014). The ubiquitination machinery of the ubiquitin system. Arabidopsis Book 12, e0174. https:// doi.org/10.1199/tab.0174.

Cerone, M.A., Londono-Vallejo, J.A., and Bacchetti, S. (2001). Telomere maintenance by telomerase and by recombination can coexist in human cells. Hum. Mol. Genet. 10, 1945-1952.

Cesare, A.J., and Griffith, J.D. (2004). Telomeric DNA in ALT cells is characterized by free telomeric circles and heterogeneous t-loops. Mol. Cell. Biol. 24, 9948-9957.

Cesare, A.J., and Reddel, R.R. (2010). Alternative lengthening of telomeres: models, mechanisms and implications. Nat. Rev. Genet. 11, 319-330. https://doi. org/10.1038/nrg2763.

Chai, W., Du, Q. Shay, J.W., and Wright, W.E. (2006). Human telomeres have different overhang sizes at leading versus lagging strands. Mol. Cell 21, 427-435.

Chapman, J.R., Taylor, M.R., and Boulton, S.J. (2012). Playing the end game: DNA double-strand break repair pathway choice. Mol. Cell 47, 497-510. https://doi. org/10.1016/j.molcel.2012.07.029.

Chen, L.Y., Redon, S., and Lingner, J. (2012). The human CST complex is a terminator of telomerase activity. Nature 488, 540-544. https://doi.org/10.1038/ nature 11269 .

Chen, Z.J., and Sun, L.J. (2009). Nonproteolytic functions of ubiquitin in cell signaling. Mol. Cell 33, 275-286. https://doi.org/10.1016/j.molcel.2009.01.014.

Chong, L., van Steensel, B., Broccoli, D., ErdjumentBromage, H., Hanish, J., Tempst, P., and de Lange, T. (1995). A human telomeric protein. Science 270, 1663-1667.

Ciechanover, A. (2005). Proteolysis: from the lysosome to ubiquitin and the proteasome. Nat. Rev. Mol. Cell Biol. 6, 79-87. https://doi.org/10.1038/nrm1552.

Ciechanover, A., and Brundin, P. (2003). The ubiquitin proteasome system in neurodegenerative diseases: sometimes the chicken, sometimes the egg. Neuron 40, 427-446.

Counter, C.M., Gupta, J., Harley, C.B., Leber, B., and Bacchetti, S. (1995). Telomerase activity in normal leukocytes and in hematologic malignancies. Blood 85, 2315-2320.

Dahlmann, B. (2007). Role of proteasomes in disease. BMC Biochem. 8 (Suppl. 1), S3.

de Bruin, D., Kantrow, S.M., Liberatore, R.A., and Zakian, V.A. (2000). Telomere folding is required for the stable maintenance of telomere position effects in yeast. Mol. Cell. Biol. 20, 7991-8000.

de la Fuente, J., and Dokal, I. (2007). Dyskeratosis congenita: advances in the understanding of the telomerase defect and the role of stem cell transplantation. Pediatr. Transplant. 11, 584-594.

de Lange, T. (2005). Shelterin: the protein complex that shapes and safeguards human telomeres. Genes Dev. 19, 2100-2110.

Diaz de Leon, A., Cronkhite, J.T., Katzenstein, A.L., Godwin, J.D., Raghu, G., Glazer, C.S., Rosenblatt, R.L., Girod, C.E., Garrity, E.R., Xing, C., et al. (2010).
Telomere lengths, pulmonary fibrosis and telomerase (TERT) mutations. PLOS ONE 5, e10680. https://doi. org/10.1371/journal.pone.0010680.

Dokal, I. (2011). Dyskeratosis congenita. Hematology Am. Soc. Hematol. Educ. Program 2011, 480-486. https:// doi.org/10.1182/asheducation-2011.1.480.

Eskelinen, E.L., and Saftig, P. (2009). Autophagy: a lysosomal degradation pathway with a central role in health and disease. Biochim. Biophys. Acta 1793, 664673. https://doi.org/10.1016/j.bbamcr.2008.07.014.

Falco, G., Lee, S.L., Stanghellini, I., Bassey, U.C., Hamatani, T., and Ko, M.S. (2007). Zscan4: a novel gene expressed exclusively in late 2-cell embryos and embryonic stem cells. Dev. Biol. 307, 539-550.

Feldser, D.M., Hackett, J.A., and Greider, C.W. (2003). Telomere dysfunction and the initiation of genome instability. Nat. Rev. Cancer 3, 623-627. https://doi. org/10.1038/nrc1142.

Fujita, K., Horikawa, I., Mondal, A.M., Jenkins, L.M., Appella, E., Vojtesek, B., Bourdon, J.C., Lane, D.P., and Harris, C.C. (2010). Positive feedback between p53 and TRF2 during telomere-damage signalling and cellular senescence. Nat. Cell Biol. 12, 1205-1212. https://doi. org/10.1038/ncb2123.

Garbe, J.C., Vrba, L., Sputova, K., Fuchs, L., Novak, P., Brothman, A.R., Jackson, M., Chin, K., LaBarge, M.A., Watts, G., et al. (2014). Immortalization of normal human mammary epithelial cells in two steps by direct targeting of senescence barriers does not require gross genomic alterations. Cell Cycle 13, 3423-3435. https:// doi.org/10.4161/15384101.2014.954456.

Gilley, D., Tanaka, H., and Herbert, B.S. (2005). Telomere dysfunction in aging and cancer. Int. J. Biochem. Cell Biol. 37, 1000-1013.

Goldberg, A.L. (2003). Protein degradation and protection against misfolded or damaged proteins. Nature 426, 895-899.

Greider, C.W. (1999). Telomeres do D-loop-T-loop. Cell 97, 419-422.

Griffith, J.D., Comeau, L., Rosenfield, S., Stansel, R.M., Bianchi, A., Moss, H., and de Lange, T. (1999). Mammalian telomeres end in a large duplex loop. Cell 97, 503-514.

Grobelny, J.V., Kulp-McEliece, M., and Broccoli, D. (2001). Effects of reconstitution of telomerase activity on telomere maintenance by the alternative lengthening of telomeres (ALT) pathway. Hum. Mol. Genet. 10, 1953-1961.

Härle-Bachor, C., and Boukamp, P. (1996). Telomerase activity in the regenerative basal layer of the epidermis inhuman skin and in immortal and carcinoma-derived skin keratinocytes. Proc. Natl. Acad. Sci. U.S.A. 93, 6476-6481.

Hayflick, L., and Moorhead, P.S. (1961). The serial cultivation of human diploid cell strains. Exp. Cell Res. 25, 585-621.

Henson, J.D., Cao, Y., Huschtscha, L.I., Chang, A.C., Au, A.Y., Pickett, H.A., and Reddel, R.R. (2009). DNA C-circles are specific and quantifiable markers of alternativelengthening-of-telomeres activity. Nat. Biotechnol. 27, 1181-1185. https://doi.org/10.1038/nbt.1587.

Her, J., Jeong, Y.Y., and Chung, I.K. (2015). PIAS1-mediated sumoylation promotes STUbL-dependent proteasomal 
degradation of the human telomeric protein TRF2. FEBS Lett. 589, 3277-3286. https://doi.org/10.1016/j. febslet.2015.09.030.

Her, Y.R., and Chung, I.K. (2009). Ubiquitin ligase RLIM modulates telomere length homeostasis through a proteolysis of TRF1. J. Biol. Chem. 284, 8557-8566. https://doi.org/10.1074/jbc.M806702200.

Hershko, A., and Ciechanover, A. (1998). The ubiquitin system. Annu. Rev. Biochem. 67, 425-479. https://doi. org/10.1146/annurev.biochem.67.1.425.

Hinkson, I.V., and Elias, J.E. (2011). The dynamic state of protein turnover: It's about time. Trends Cell Biol. 21, 293-303. https://doi.org/10.1016/j.tcb.2011.02.002.

Hirata, T., Amano, T., Nakatake, Y., Amano, M., Piao, Y., Hoang, H.G., and Ko, M.S. (2012). Zscan 4 transiently reactivates early embryonic genes during the generation of induced pluripotent stem cells. Sci. Rep. 2, 208. https://doi.org/10.1038/srep00208.

Hockemeyer, D., Sfeir, A.J., Shay, J.W., Wright, W.E., and de Lange, T. (2005). POT1 protects telomeres from a transient DNA damage response and determines how human chromosomes end. EMBO J. 24, 2667-2678.

Houghtaling, B.R., Cuttonaro, L., Chang, W., and Smith, S. (2004). A dynamic molecular link between the telomere length regulator TRF1 and the chromosome end protector TRF2. Curr. Biol. 14, 1621-1631. https://doi. org/10.1016/j.cub.2004.08.052.

Hu, J., Hwang, S.S., Liesa, M., Gan, B., Sahin, E., Jaskelioff, M., Ding, Z., Ying, H., Boutin, A.T., Zhang, H., et al. (2012). Antitelomerase therapy provokes ALT and mitochondrial adaptive mechanisms in cancer. Cell 148, 651-663. https://doi.org/10.1016/j.cell.2011.12.028.

Hu, Y., Shi, G., Zhang, L., Li, F., Jiang, Y., Jiang, S., Ma, W., Zhao, Y., Songyang, Z., and Huang, J. (2016). Switch telomerase to ALT mechanism by inducing telomeric DNA damages and dysfunction of ATRX and DAXX. Sci. Rep. 6, 32280. https://doi.org/10.1038/srep32280.

Huang, Q. and Figueiredo-Pereira, M.E. (2010). Ubiquitin/ proteasome pathway impairment in neurodegeneration: therapeutic implications. Apoptosis 15, 1292-1311. https://doi.org/10.1007/s10495-010-0466-z.

Huen, M.S., Grant, R., Manke, I., Minn, K., Yu, X., Yaffe, M.B., and Chen, J. (2007). RNF8 transduces the DNAdamage signal via histone ubiquitylation and checkpoint protein assembly. Cell 131, 901-914.

Jackson, S.P., and Bartek, J. (2009). The DNA-damage response in human biology and disease. Nature 461, 1071-1078. https://doi.org/10.1038/nature08467.

Jemal, A., Ward, E.M., Johnson, C.J., Cronin, K.A., Ma, J., Ryerson, B., Mariotto, A., Lake, A.J., Wilson, R., Sherman, R.L., et al. (2017). Annual report to the nation on the status of cancer, 1975-2014, Featuring Survival. J. Natl. Cancer Inst. 109, . https://doi.org/10.1093/jnci/ djx030.

Jiang, J., Lv, W., Ye, X., Wang, L., Zhang, M., Yang, H., Okuka, M., Zhou, C., Zhang, X., Liu, L., et al. (2013). Zscan4 promotes genomic stability during reprogramming and dramatically improves the quality of iPS cells as demonstrated by tetraploid complementation. Cell Res. 23, 92-106. https://doi.org/10.1038/cr.2012.157.

Jonkers, J. (2012). Tracking evolution of BRCA1-associated breast cancer. Cancer Discov. 2, 486-488. https://doi. org/10.1158/2159-8290.CD-12-0186.
Karlseder, J., Broccoli, D., Dai, Y., Hardy, S., and de Lange, T. (1999). p53- and ATM-dependent apoptosis induced by telomeres lacking TRF2. Science 283, 1321-1325.

Karlseder, J., Hoke, K., Mirzoeva, O.K., Bakkenist, C., Kastan, M.B., Petrini, J.H., and de Lange, T. (2004). The telomeric protein TRF2 binds the ATM kinase and can inhibit the ATM-dependent DNA damage response. PLOS Biol. 2, E240. https://doi.org/10.1371/journal. pbio.0020240.

Kelleher, C., Kurth, I., and Lingner, J. (2005). Human protection of telomeres 1 (POT1) is a negative regulator of telomerase activity in vitro. Mol. Cell. Biol. 25, 808-818.

Kim, J., and Chung, I.K. (2014). The splicing factor U2AF65 stabilizes TRF1 protein by inhibiting its ubiquitindependent proteolysis. Biochem. Biophys. Res. Commun. 443, 1124-1130. https://doi.org/10.1016/j. bbrc.2013.12.118.

Kim, S.H., Kaminker, P., and Campisi, J. (1999). TIN2, a new regulator of telomere length in human cells. Nat. Genet. 23, 405-412. https://doi.org/10.1038/70508.

Kim, S.H., Han, S., You, Y.H., Chen, D.J., and Campisi, J. (2003). The human telomere-associated protein TIN2 stimulates interactions between telomeric DNA tracts in vitro. EMBO reports 4, 685-691.

Kim, S.H., Beausejour, C., Davalos, A.R., Kaminker, P., Heo, S.J., and Campisi, J. (2004). TIN2 mediates functions of TRF2 at human telomeres. J. Biol. Chem. 279, 4379943804. https://doi.org/10.1074/jbc.M408650200.

Korolchuk, V.I., Menzies, F.M., and Rubinsztein, D.C. (2010). Mechanisms of cross-talk between the ubiquitin-proteasome and autophagy-lysosome systems. FEBS Lett. 584, 1393-1398. https://doi.org/10.1016/j. febslet.2009.12.047.

Kunická, Z., Mucha, I., and Fajkus, J. (2008). Telomerase activity in head and neck cancer. Anticancer Res. 28, 3125-3129.

Lee, K., and Gollahon, L.S. (2014). Zscan4 interacts directly with human Rap1 in cancer cells regardless of telomerase status. Cancer Biol. Ther. 15, 1094-1105. https://doi. org/10.4161/cbt.29220.

Lee, K., and Gollahon, L.S. (2015). ZSCAN4 and TRF1: A functionally indirect interaction in cancer cells independent of telomerase activity. Biochem. Biophys. Res. Commun. 466, 644-649. https://doi. org/10.1016/j.bbrc.2015.09.107.

Lee, T.H., Perrem, K., Harper, J.W., Lu, K.P., and Zhou, X.Z. (2006). The F-box protein FBX4 targets PIN2/ TRF1 for ubiquitin-mediated degradation and regulates telomere maintenance. J. Biol. Chem. 281, 759-768.

Lei, M., Zaug, A.J., Podell, E.R., and Cech, T.R. (2005). Switching human telomerase on and off with hPOT1 protein in vitro. J. Biol. Chem. 280, 20449-20456.

Lescasse, R., Pobiega, S., Callebaut, I., and Marcand, S. (2013). End-joining inhibition at telomeres requires the translocase and polySUMO-dependent ubiquitin ligase Uls1. EMBO J. 32, 805-815. https://doi.org/10.1038/ emboj.2013.24.

Li, B., Oestreich, S., and de Lange, T. (2000). Identification of human Rap1: implications for telomere evolution. Cell 101, 471-483.

Liu, D., Safari, A., O’Connor, M.S., Chan, D.W., Laegeler, A., Qin, J., and Songyang, Z. (2004). PTOP interacts 
with POT1 and regulates its localization to telomeres. Nat. Cell Biol. 6, 673-680. https://doi.org/10.1038/ ncb1142.

Loayza, D., and De Lange, T. (2003). POT1 as a terminal transducer of TRF1 telomere length control. Nature 423, 1013-1018. https://doi.org/10.1038/nature01688.

Makarov, V.L., Hirose, Y., and Langmore, J.P. (1997). Long $\mathrm{G}$ tails at both ends of human chromosomes suggest a C strand degradation mechanism for telomere shortening. Cell 88, 657-666.

McElligott, R., and Wellinger, R.J. (1997). The terminal DNA structure of mammalian chromosomes. EMBO J. 16, 3705-3714. https://doi.org/10.1093/ emboj/16.12.3705.

Miyagawa, K., Low, R.S., Santosa, V., Tsuji, H., Moser, B.A., Fujisawa, S., Harland, J.L., Raguimova, O.N., Go, A., Ueno, M., et al. (2014). SUMOylation regulates telomere length by targeting the shelterin subunit Tpz1(Tpp1) to modulate shelterin-Stn 1 interaction in fission yeast. Proc. Natl. Acad. Sci. U.S.A. 111, 5950-5955. https:// doi.org/10.1073/pnas.1401359111.

Morreale, F.E., and Walden, H. (2016). Types of Ubiquitin Ligases. Cell 165, 248-248.e1.

Muñoz-Jordán, J.L., Cross, G.A., de Lange, T., and Griffith, J.D. (2001). t-loops at trypanosome telomeres. EMBO J. 20, 579-588. https://doi.org/10.1093/ emboj/20.3.579.

Murti, K.G., and Prescott, D.M. (1999). Telomeres of polytene chromosomes in a ciliated protozoan terminate in duplex DNA loops. Proc. Natl. Acad. Sci. U.S.A. 96, 14436-14439.

Nandi, D., Tahiliani, P., Kumar, A., and Chandu, D. (2006). The ubiquitin-proteasome system. J. Biosci. 31, 137-155.

Nelson, N.D., and Bertuch, A.A. (2012). Dyskeratosis congenita as a disorder of telomere maintenance. Mutat. Res. 730, 43-51. https://doi.org/10.1016/j. mrfmmm.2011.06.008.

Ohta, T., and Fukuda, M. (2004). Ubiquitin and breast cancer. Oncogene 23, 2079-2088.

O’Sullivan, R.J., and Karlseder, J. (2010). Telomeres: protecting chromosomes against genome instability. Nat. Rev. Mol. Cell Biol. 11, 171-181. https://doi. org $/ 10.1038 / \mathrm{nrm} 2848$.

Ozkan, E., Yu, H., and Deisenhofer, J. (2005). Mechanistic insight into the allosteric activation of a ubiquitinconjugating enzyme by RING-type ubiquitin ligases. Proc. Natl. Acad. Sci. U.S.A. 102, 18890-18895.

Park, H.S., Hwang, I., Choi, K.A., Jeong, H., Lee, J.Y., and Hong, S. (2015). Generation of induced pluripotent stem cells without genetic defects by small molecules. Biomaterials 39, 47-58. https://doi.org/10.1016/j. biomaterials.2014.10.055.

Perrem, K., Colgin, L.M., Neumann, A.A., Yeager, T.R., and Reddel, R.R. (2001). Coexistence of alternative lengthening of telomeres and telomerase in hTERTtransfected GM847 cells. Mol. Cell. Biol. 21, 3862-3875. https://doi.org/10.1128/MCB.21.12.3862-3875.2001.

Peuscher, M.H., and Jacobs, J.J. (2011). DNA-damage response and repair activities at uncapped telomeres depend on RNF8. Nat. Cell Biol. 13, 1139-1145. https://doi.org/10.1038/ncb2326.
Pickart, C.M., and Eddins, M.J. (2004). Ubiquitin: structures, functions, mechanisms. Biochim. Biophys. Acta 1695, 55-72.

Portney, B.A., Khatri, R., Meltzer, W.A., Mariano, J.M., and Zalzman, M. (2018). ZSCAN4 is negatively regulated by the ubiquitin-proteasome system and the E3 ubiquitin ligase RNF20. Biochem. Biophys. Res. Commun. 498, 72-78.

Rai, R., Li, J.M., Zheng, H., Lok, G.T., Deng, Y., Huen, M.S., Chen, J., Jin, J., and Chang, S. (2011). The E3 ubiquitin ligase Rnf8 stabilizes Tpp1 to promote telomere end protection. Nat. Struct. Mol. Biol. 18, 1400-1407. https://doi.org/10.1038/nsmb.2172.

Rai, R., Chen, Y., Lei, M., and Chang, S. (2016). TRF2RAP1 is required to protect telomeres from engaging in homologous recombination-mediated deletions and fusions. Nat. Commun. 7, 10881. https://doi. org/10.1038/ncomms 10881 .

Reinstein, E., and Ciechanover, A. (2006). Narrative review: protein degradation and human diseases: the ubiquitin connection. Ann. Intern. Med. 145, 676-684.

Samassekou, O., Gadji, M., Drouin, R., and Yan, J. (2010). Sizing the ends: normal length of human telomeres. Ann. Anat. 192, 284-291. https://doi.org/10.1016/j. aanat.2010.07.005.

Savage, S.A., and Alter, B.P. (2009). Dyskeratosis congenita. Hematol. Oncol. Clin. North Am. 23, 215-231. https:// doi.org/10.1016/j.hoc.2009.01.003.

Schieker, M., Gülkan, H., Austrup, B., Neth, P., and Mutschler, W. (2004). [Telomerase activity and telomere length of human mesenchymal stem cells. Changes during osteogenic differentiation.] Orthopade 33, 1373-1377. https://doi.org/10.1007/s00132-0040739-8.

Smith, J.L., Lee, L.C., Read, A., Li, Q. Yu, B., Lee, C.S., and Luo, J. (2016). One-step immortalization of primary human airway epithelial cells capable of oncogenic transformation. Cell Biosci. 6, 57. https://doi. org/10.1186/s13578-016-0122-6.

Stansel, R.M., de Lange, T., and Griffith, J.D. (2001). T-loop assembly in vitro involves binding of TRF2 near the 3' telomeric overhang. EMBO J. 20, 5532-5540. https:// doi.org/10.1093/emboj/20.19.5532.

Sugimoto, M., Tahara, H., Ide, T., and Furuichi, Y. (2004). Steps involved in immortalization and tumorigenesis in human B-lymphoblastoid cell lines transformed by Epstein-Barr virus. Cancer Res. 64, 3361-3364. https:// doi.org/10.1158/0008-5472.CAN-04-0079.

Sun, Z.W., and Allis, C.D. (2002). Ubiquitination of histone $\mathrm{H} 2 \mathrm{~B}$ regulates $\mathrm{H} 3$ methylation and gene silencing in yeast. Nature 418, 104-108. https://doi.org/10.1038/ nature00883.

Tanaka, K., Suzuki, T., Hattori, N., and Mizuno, Y. (2004). Ubiquitin, proteasome and parkin. Biochim. Biophys. Acta 1695, 235-247.

Vaziri, H., and Benchimol, S. (1996). From telomere loss to p53 induction and activation of a DNA-damage pathway at senescence: the telomere loss/DNA damage model of cell aging. Exp. Gerontol. 31, 295-301.

Vaziri, H., Dragowska, W., Allsopp, R.C., Thomas, T.E., Harley, C.B., and Lansdorp, P.M. (1994). Evidence for a mitotic clock in human hematopoietic stem cells: loss 
of telomeric DNA with age. Proc. Natl. Acad. Sci. U.S.A. 91, 9857-9860.

Wang, C., Xiao, H., Ma, J., Zhu, Y., Yu, J., Sun, L., Sun, H., Liu, Y., Jin, C., and Huang, H. (2013). The F-box protein $\beta$-TrCP promotes ubiquitination of TRF 1 and regulates the ALT-associated PML bodies formation in U2OS cells. Biochem. Biophys. Res. Commun. 434, 728-734. https://doi.org/10.1016/j.bbrc.2013.03.096.

Yang, Z., and Klionsky, D.J. (2010). Mammalian autophagy: core molecular machinery and signaling regulation. Curr. Opin. Cell Biol. 22, 124-131. https://doi. org/10.1016/j.ceb.2009.11.014.

Ye, J.Z., and de Lange, T. (2004). TIN2 is a tankyrase 1 PARP modulator in the TRF1 telomere length control complex. Nat. Genet. 36, 618-623. https://doi. org/10.1038/ng1360.

Ye, Y., and Rape, M. (2009). Building ubiquitin chains: E2 enzymes at work. Nat. Rev. Mol. Cell Biol. 10, 755-764. https://doi.org/10.1038/nrm2780.

Yeager, T.R., Neumann, A.A., Englezou, A., Huschtscha, L.I., Noble, J.R., and Reddel, R.R. (1999). Telomerasenegative immortalized human cells contain a novel type of promyelocytic leukemia (PML) body. Cancer Res. 59, 4175-4179.

Yu, T.Y., Kao, Y.W., and Lin, J.J. (2014). Telomeric transcripts stimulate telomere recombination to suppress senescence in cells lacking telomerase. Proc. Natl. Acad. Sci. U.S.A. 111, 3377-3382. https://doi.org/10.1073/ pnas.1307415111.

Zalzman, M., Falco, G., Sharova, L.V., Nishiyama, A., Thomas, M., Lee, S.L., Stagg, C.A., Hoang, H.G., Yang, H.T., Indig, F.E., et al. (2010). Zscan4 regulates telomere elongation and genomic stability in ES cells. Nature 464, 858-863. https://doi.org/10.1038/nature08882.

Zemp, I., and Lingner, J. (2014). The shelterin component TPP1 is a binding partner and substrate for the deubiquitinating enzyme USP7. J. Biol. Chem. 289, 28595-28606. https://doi.org/10.1074/jbc. M114.596056.

Zhong, Z., Shiue, L., Kaplan, S., and de Lange, T. (1992). A mammalian factor that binds telomeric TTAGGG repeats in vitro. Mol. Cell. Biol. 12, 4834-4843.

Zhong, Z.H., Jiang, W.Q. Cesare, A.J., Neumann, A.A., Wadhwa, R., and Reddel, R.R. (2007). Disruption of telomere maintenance by depletion of the MRE11/ RAD50/NBS1 complex in cells that use alternative lengthening of telomeres. J. Biol. Chem. 282, 2931429322.

Zhou, P. (2004). Determining protein half-lives. Methods Mol. Biol. 284, 67-77. 
